\title{
Transparencia operativa de las comunidades autónomas españolas mediante sus portales de datos abiertos
}

\section{Operational transparency of the Spanish autonomous communities through their open data portals}

\author{
Ricardo Curto-Rodríguez
}

Cómo citar este artículo:

Curto-Rodríguez, Ricardo (2020). "Transparencia operativa de las comunidades autónomas españolas mediante sus portales de datos abiertos". El profesional de la información, v. 29, n. 1, e290115.

https://doi.org/10.3145/epi.2020.ene.15

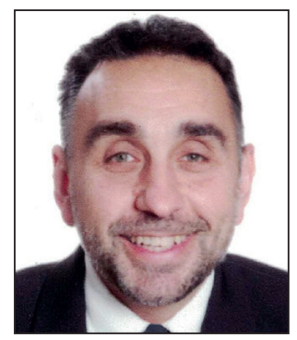

\author{
Ricardo Curto-Rodríguez \\ https://orcid.org/0000-0002-6628-5278 \\ Universidad de Oviedo \\ Facultad de Economía y Empresa \\ Dep. de Comercialización e Investigación de Mercados \\ Avenida del Cristo, s/n. 33006 Oviedo, España \\ curtoricardo@uniovi.es
}

\section{Resumen}

Una vez consolidadas a nivel mundial las iniciativas de datos abiertos, nos parece relevante averiguar si los portales autonómicos españoles tienen utilidad para la transparencia activa, elaborando un indicador en tres períodos de tiempo: 2013, 2015 y 2018. Este indicador valora tanto la existencia de información como su calidad reutilizadora. Tras valorar los resultados obtenidos, se indaga sobre la existencia de grupos diferenciados de autonomías empleando, cuando es pertinente, un análisis clúster. Los análisis efectuados encuentran indicios de que hay dos conglomerados de autonomías: avanzadas y rezagadas, hallazgos en línea con lo planteado por dos teorías contrapuestas sobre la divulgación de información del sector público: agencia y neoinstitucional.

\section{Palabras clave}

Análisis clúster; Transparencia; Rendición de cuentas; Datos; Datos abiertos; Información pública; Comunidades autónomas; España.

\begin{abstract}
Once open data initiatives are consolidated worldwide, it is important to find out if the Spanish regional open data portals are useful for active transparency. To this end, we developed an indicator to assess the existence of information and its reusable quality in three periods of time (2013, 2015 and 2018). Then, we inquired about the existence of differentiated groups of regional governments using, when necessary, cluster analysis. We found indications that there are two conglomerates of governments: advanced and lagged. These findings are in line with those offered by two opposing views on the dissemination of information from the public sector: agency and neo-institutional theories.
\end{abstract}

\section{Keywords}

Cluster analysis; Transparency; Accountability; Data; Open data; Public information; Regional governments; Spain.

\section{Introducción: movimiento de portales open data y comunidades autónomas españolas}

Pocos meses después del discurso inaugural del presidente de Estados Unidos Barack Obama, en el que prometió una administración transparente con la ciudadanía (Ferrer-Sapena; Peset; Aleixandre-Benavent, 2011), el 21 de mayo de 2009, se produjo el lanzamiento de data.gov, el portal estadounidense de datos abiertos, con 76 conjuntos de datos alojados. Diez años después y con un incremento que le hace llegar hasta los 232.000 conjuntos de datos (datasets), se mantiene como el mejor ejemplo para las más de 2.600 iniciativas similares a nivel mundial identificadas en la lista Open data inception (Open Data Soft, 2019) (figura 1). 


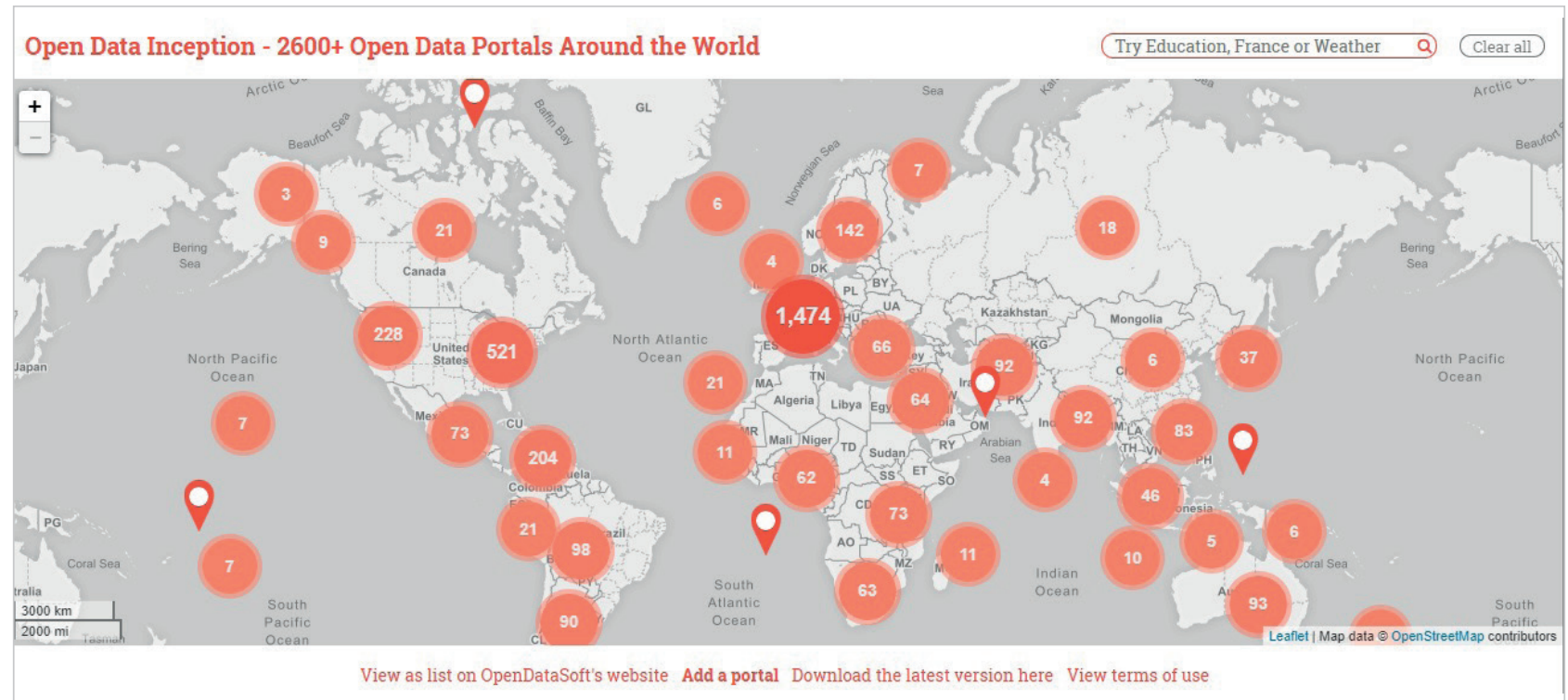

Figura 1. Portales de datos abiertos según Open data inception. Fuente: Open data soft https://opendatainception.io

Según dicho estudio, existen 361 portales de datos abiertos en España de hospitales, universidades, ministerios, ayuntamientos, diputaciones, autonomías y gobierno central. La iniciativa estatal (datos.gob.es), que federa o sindica información de la mayor parte de los portales del país, localiza 295 iniciativas (224 de la administración local, 43 de la administración del Estado, 18 de la autonómica y 10 de universidades).

De los 18 portales dependientes de comunidades autónomas (CCAA), 15 corresponden a repositorios analizados por nuestro estudio. La tabla 1 recoge nombre y número de conjuntos de datos ofertados a finales de cada uno de los años referidos. Se excluyen: Datos abiertos del Instituto Cántabro de Estadística (Istac) y Datos abiertos de la Comunidad de Madrid $^{2}$.

Tabla 1. Portales de datos abiertos autonómicos y conjuntos de datos albergados

\begin{tabular}{|c|c|c|c|c|}
\hline Comunidad autónoma & Nombre del portal & Datasets 2013 & Datasets 2015 & Datasets 2017 \\
\hline Andalucía & Datos abiertos & 70 & 178 & 464 \\
\hline Aragón & Aragón open data & 440 & 2.519 & 2.794 \\
\hline Canarias & Datos abiertos Canarias & Sin portal & 45 & 43 \\
\hline Cantabria & & Sin portal & Sin portal & Sin portal \\
\hline Castilla y León & Datos abiertos de Castilla y León & 124 & 176 & 334 \\
\hline Castilla-La Mancha & Datos abiertos de Castilla-La Mancha & 42 & 59 & 207 \\
\hline Cataluña & Dades obertes GenCat & 1.460 & 1.468 & 571 \\
\hline Comunidad de Madrid & & Sin portal & Sin portal & Sin portal \\
\hline Com. Foral de Navarra & Open data & 135 & 190 & 1.186 \\
\hline Comunidad Valenciana & Dades obertes GVA & Sin portal & 279 & 338 \\
\hline Extremadura & Gobierno abierto - Catálogo de datos & 26 & 25 & 25 \\
\hline Galicia & abert@s & 292 & 324 & 349 \\
\hline Islas Baleares & Dades obertes $C A I B$ & 42 & 42 & 42 \\
\hline La Rioja & Datos abiertos Rioja & 64 & 86 & 265 \\
\hline País Vasco & Opendataeuskadi & 2.280 & 2.242 & 4.123 \\
\hline Principado de Asturias & Open data del Principado de Asturias & Sin portal & 419 & 42 \\
\hline Región de Murcia & Datos abiertos Región de Murcia & Sin portal & Sin portal & 271 \\
\hline
\end{tabular}

Hemos decidido circunscribir nuestra investigación de los portales de datos abiertos a un ámbito poco contemplado en la bibliografía, las CCAA, que tienen una importancia indiscutible en la organización territorial de un país cuasifederal (Bastida et al., 2019) fuertemente descentralizado como España (Martínez-Vázquez, 2014). No en vano las autonomías tienen competencias transferidas entre las que destacan sanidad y educación (Fernández-Llera; Morán-Méndez, 2013), que son las partidas a las que más recursos se le dedican según muestra la figura 2. 
España, referente de buenas prácticas en datos abiertos $y$ uno de los líderes europeos en 2018 junto con Irlanda y Francia, según el estudio Open data best practices in Europe's top three performers (European data portal, 2018), ha adoptado una posición activa de seguimiento de las directrices de datos abiertos que tuvieron su origen en los ocho principios de Open government data ${ }^{3}$ (Sunlight Foundation, 2007): datos completos, primarios, actualizados, accesibles, procesables, no discriminatorios, no propietarios y de licencia libre. Estas recomendaciones siguen vigentes en la actualidad y han servido de base para que otros organismos añadieran otras como derecho ciudadano, financiación pública, integridad, privacidad y confidencialidad, y preservación (Curto-Rodríguez, 2017).

Al movimiento de datos abiertos se le atribuyen varias utilidades como la creación de valor, el incremento de la interoperabilidad o la mejora de la eficiencia interna de las administraciones. No obstante, queremos enfocar en este estudio su papel para el fomento de la transparencia ${ }^{4}$, puesto que los datos abiertos han generado muchas expectativas al respecto (Ferrer-Sapena; Sánchez-Pérez, 2013). Esta iniciativa podría aprovecharse como un excelente mecanismo de rendición de cuentas al facilitar la reutilización de la información ofertada, máxime cuando otras posibilidades como los portales de transparencia regionales o la web autonómica están publicando datos básicamente en formatos pdf o html, de difícil manejo (Curto-Rodríguez, 2016).

Vivimos en la era de los datos, siendo los gobiernos uno de sus principales creadores (Cerrillo-Martínez, 2018). Los datos abiertos se han convertido en un signo inequívoco de una administración transparente y responsable (Höchtl, 2012), siendo la base de la toma de decisiones y la materia prima para la rendición de cuentas. Sin datos de alta calidad, diseñar, monitorear y evaluar políticas efectivas se vuelve casi imposible (European Commission, 2014, p. 4).

Obviamente, la información debe poder usarse (Ballester-Espinosa, 2015), y a tal efecto es muy conveniente que sea depositada en la Web para favorecer las relaciones entre datos y la creación de redes (García-García, 2013), siendo datos abiertos la mejor iniciativa que lo permite. Los datos abiertos facilitan la reutilización al ser fácilmente localizables, reutilizables y no estár sometidos a restricciones técnicas o jurídicas (Cerrillo-Martínez, 2014), por lo que esperamos que su potencial de análisis, su facilidad de cruce con otra información y/o la elaboración de aplicaciones sean aprovechadas por cualquier interesado en base a los principios de crowdsourcing de Surowiecki (2005) y The many minds principle de Walsh y Pollock (2007).

No está claro que todos los gobiernos deseen suministrar información relacionada con la transparencia activa, según se deduce del postulado de dos teorías contrapuestas de la divulgación de las administraciones públicas.

La primera es la teoría de la agencia, la más empleada en la bibliografía (Rodríguez-Bolívar; Alcaide-Muñoz; López-Hernández, 2013). Esta teoría es aplicable cuando los ciudadanos (principal) delegan cierta toma de decisiones en los gobiernos (agentes), requiriendo para mayor confianza en los actores políticos, que se revele información que permita su monitoreo reduciendo la asimetría de información (Laswad; Fisher; Oyelere, 2005), limitando los conflictos entre ciudadanos y gobernantes (Zimmerman, 1977), y resolviendo el problema de la agencia (Ferejohn, 1999).

La segunda, la teoría neoinstitucional, aborda un estudio sociológico de las instituciones, entendidas como espacios donde los actores sociales desenvuelven sus prácticas. Esa teoría es consciente de la réplica de iniciativas asociadas con la transparencia activa, como los portales de datos abiertos o de transparencia (García-García; Alonso-Magdaleno; Alonso-Magdaleno, 2016; Curto-Rodríguez, 2019a), que contribuyen a una mayor reputación gubernamental, a una apariencia de modernidad y a un posicionamiento como administración pública avanzada. No obstante, la puesta en marcha de un repositorio podría convertirse en un fin en sí mismo siendo posible no suministrar información "transparente" y conformarse con imitar iniciativas favorecedoras de una buena opinión pública sin dotarlas de contenido.

Estas teorías implican, como indica Curto-Rodríguez (2019b), que podemos esperar dos tipos de comportamientos fundamentales: 
- en línea con la teoría neoinstitucional, la puesta en marcha de un portal de datos abiertos permitiría que los gobiernos cumplieran su objetivo de ganar valor frente a sus rivales políticos, pudiendo incurrir en un comportamiento de transparencia aparente publicando información irrelevante respecto al control de su gestión;

- en el extremo opuesto, estaría el comportamiento de transparencia sincera que, conforme a lo propuesto por la teoría de la agencia y motivado por el deseo de ampliar el conocimiento ciudadano, haría que fueran incorporados al portal múltiples conjuntos de datos de transparencia activa para justificar el desempeño gubernamental.

Fijamos el objetivo de nuestra investigación en medir la información transparente alojada en los portales de datos abiertos y su potencial a efectos de reutilización. Por ello, el siguiente apartado, además de sugerir comportamientos esperados, elabora y justifica el indicador empleado. El trabajo continúa con una tercera sección destinada a presentar las técnicas estadísticas utilizadas (análisis descriptivo y clúster), seguida de la presentación de los resultados, las conclusiones y las futuras líneas de investigación abordables.

\section{Transparencia activa: lista de comprobación, herramientas de valoración, calidad reutilizadora e indicador de transparencia datos abiertos}

Transparencia, buen gobierno y rendición de cuentas son temas de actualidad que se recogen en las grandes líneas de las políticas públicas y que comprenden comunicaciones, reglamentos, memorandos, guías, normas técnicas, directivas, leyes, apertura de repositorios digitales, etc. ${ }^{5}$, formando parte del debate social como una piedra angular de todos los discursos políticos (Delgado-Morales; López-Carvajal; Sierra-Rodríguez, 2015) y convirtiéndose poco a poco en una realidad (Sierra-Rodríguez, 2018a, p. 27).

Según Alt, Lassen y Rose (2006), la transparencia ha sido una parte integral de los intentos de reformar la gobernanza del sector público siendo muchos los gobiernos que han trabajado para aumentar la apertura y la transparencia en sus acciones (Bertot; Jaeger; Grimes, 2010), pues ya son más de 100 los países que cuentan con leyes que regulan dicho cometido.

En nuestro país ha habido una expansión del interés sobre estas materias tras la publicación de la Ley 19/2013 de transparencia, acceso a la información pública y buen gobierno (Sierra-Rodríguez, 2018b). Antes de la aprobación de dicha normativa, España carecía de una ley ad hoc sobre transparencia o derecho de acceso a la información (Beltrán-Orenes; Martínez-Pastor, 2016). Ha sido esta ley, que responde a los compromisos adquiridos con la alianza con el gobierno abierto (Cotino-Hueso, 2015; Beltrán-Orenes; Martínez-Pastor, 2017), lo que ha permitido a nuestro país dejar de ser el único miembro del Consejo de Europa con una población de más de un millón de habitantes que no disponía de una ley específica de transparencia y acceso a la información pública (ViIloria, 2014), lo cual era consecuencia de la inercia histórica, política y social del entorno (Cotino-Hueso, 2014).

Una bondad evidente de la transparencia en la administración pública es contribuir a la calidad de vida de los ciudadanos y mejorar la confianza en el gobierno debido a su valor informativo y su función de monitoreo (Galli; Rizzo; Scaglioni, 2019, p. 97), permitiendo el escrutinio y el control de la administración (Boix, 2015, p. 123, Graells-Costa, 2013), conteniendo un gran poder de transformación social y político (Delgado-Morales, 2018), y convirtiéndose en el mejor antídoto contra la corrupción (Abu-Bakar; Saleh, 2011).

$Y$ si bien es cierto que entendemos que la transparencia no es unidireccional y no está vinculada exclusivamente a la proactividad de la administración en cuanto a la divulgación por defecto, puesto que el derecho de acceso a la información pública forma parte de este concepto, esta investigación concentra sus esfuerzos en medir el grado y calidad a efectos de reutilización de los datos suministrados en los portales de datos abiertos autonómicos. En base a los resultados alcanzados, estaremos en condiciones de sugerir si el comportamiento de las CCAA se asocia más con un modelo de agencia o con un modelo institucional. Dicho de otro modo, podemos valorar si las autonomías reducen las asimetrías informativas divulgando información transparente siendo avanzadas o si se quedan rezagadas (o al margen de este proceso) conformándose simplemente con aperturar el portal.

Tabla 2. Lista de comprobación

\begin{tabular}{|l|}
\hline Rendición de cuentas económico-financiera \\
\hline Presupuestos de gastos e ingresos: \\
\hline Por cualquier clasificación (orgánica, funcional o económica) \\
\hline Por partidas presupuestarias \\
\hline Memoria con descripción de los programas presupuestarios \\
\hline Ejecución presupuestaria: \\
\hline Información mensual de ejecución \\
\hline Liquidación anual \\
\hline Modificaciones presupuestarias \\
\hline Presupuestos de entes, sociedades y fundaciones públicas \\
\hline Cuentas anuales de empresas públicas \\
\hline Cuenta general de la Comunidad Autónoma \\
\hline Cumplimiento del objetivo de estabilidad presupuestaria \\
\hline Nivel y desglose de endeudamiento \\
\hline Informe de fiscalización \\
\hline Rendición de cuentas de los gobernantes: \\
\hline Organigrama y contactos \\
\hline Retribuciones a miembros del gobierno y altos cargos \\
\hline Declaraciones de bienes de los miembros del gobierno \\
\hline Declaraciones de actividades de miembros del gobierno \\
\hline Lista y retribuciones de cargos de confianza \\
\hline Rendición de cuentas en las ayudas y contrataciones: \\
\hline Ayudas y subvenciones \\
\hline Becas, premios o concursos \\
\hline Contratos adjudicados \\
\hline
\end{tabular}


Con el cometido de cuantificar divulgación efectuada, nos hemos servido de una lista de comprobación propia compuesta por veinte items, distribuidos en tres apartados: existencia de datos económico-financieros, información de los gobernantes, y ayudas y subvenciones (tabla 2), siendo conscientes de la subjetividad inherente en su formulación. Pero como bien indica Cerrillo-Martínez (2012), la bibliografía ha aportado tantas definiciones de transparencia como intentos ha habido para definirla, lo que muestra la complejidad en la medición del concepto (Cuadrado-Ballesteros, 2014) y su controvertida cuantificación (Ma; Wu, 2011).

Esta check list ha sido utilizada en varias investigaciones ${ }^{6}$ y, aun siendo previa al estudio publicado por la Federación Española de Municipios y Provincias sobre los 40 conjuntos de datos a publicar por las entidades locales (FEMP, 2019), comparte algunos apartados. Consideramos que se trata de una lista completa que permite valorar la divulgación de información a efectos de la supervisión de la gestión de asuntos públicos, y que no adolece del aprendizaje perverso señalado por Alonso-Magdaleno y García-García (2014) sí presente en el Índice de las Comunidades Autónomas que elabora Transparencia Internacional España, en cuanto a que los dirigentes van a concentrar esfuerzos en aquello que es evaluado por el índice (que además es requerido con anterioridad).

No obstante, para conseguir realmente empoderar al ciudadano, la puesta a disposición de la información online debe cumplir unos criterios de accesibilidad y usabilidad para que sea fácilmente localizable y pueda ser reutilizada (Martínez-Moya, 2015).

Hay varios indicadores que contemplan los estándares técnicos en los que se suministra el dato, como Meloda, descrito en Abella, Ortiz-de-Urbina-Criado y De-Pablos-Heredero (2014) y Abella (2016), y utilizado en Abella, Ortiz-de-Urbina-Criado y De-Pablos-Heredero (2018) y en Vicente-Paños y Jordán Alfonso (2017), si bien preferimos continuar la línea de investigación de García-García y Curto-Rodríguez (2018; 2019a; 2019b) que valoran el formato en el que la información está disponible según Tim Berners-Lee, el creador de la Web.

Los estándares técnicos son atendidos por ambos indicadores en función del formato en que la información viene suministrada (si bien con distintas escalas). Aunque Meloda considera otros apartados adicionales, hemos preferido el empleo de un indicador sencillo y fácilmente comprensible por todos los interesados, ampliamente avalado y con mayor utilización en la investigación académica.

Así pues, únicamente se medirá la capacidad reutilizadora según el modelo cinco estrellas ${ }^{7}$ de Tim Berners-Lee (tabla 3), de forma que cada ítem atendido (con conjuntos de datos que contengan información completa y actualizada de cada requerimiento) recibirá un punto adicional por cada uno de los cuatro primeros niveles o estrellas que alcance . $^{8}$

Tabla 3. Esquema 5 star open data

\begin{tabular}{|c|l|}
\hline \multicolumn{1}{|c|}{} & Cualquier información en la web en formato no estructurado. Ejemplo: formato pdf \\
\hline & Datos legibles por máquina, pero en formato propietario. Ejemplo: formato xls \\
\hline & Además de formato estructurado, abiertos y no propietarios. Ejemplo: formato csv \\
\hline & Datos vinculables y reutilizables. Ejemplo: formato rdf \\
\hline & Nivel de datos enlazados que permite enlazar los datos alojados con otros creando un nuevo contexto \\
\hline
\end{tabular}

Fuente: Berners-Lee (2010)

En base a lo expuesto, ya podemos formular nuestro indicador de transparencia datos abiertos (TOD), cuya valoración oscilará entre los cero puntos si las autonomías no disponen de portal de datos abiertos o carecen de la información buscada por la lista de comprobación, y los 100 puntos si consiguieran completar los 20 items en formato/s nivel cuatro estrellas, con lo que damos paso a la discusión metodológica.

\section{Análisis clúster: marco teórico}

El análisis clúster o de conglomerados busca identificar grupos de sujetos lo más homogéneos posible entre sí y lo más heterogéneos posibles respecto a los individuos del resto de grupos (Valderrey, 2010, p. 359). Nuestra investigación va a emplear como variable discriminadora la puntuación alcanzada por las CCAA en el indicador TOD y, si bien es cierto que cuando el conjunto a clasificar está descrito por una sola variable, la obtención de grupos es aparentemente sencilla (Grande-Esteban; Abascal-Fernández, 2007, p. 397), la aplicación de la técnica consigue una división lo más aséptica posible eliminando susceptibilidades y minimizando el sesgo del investigador.

En primer lugar, utilizaremos una técnica de tipo jerárquico, recomendable cuando existen pocas observaciones (Trespalacios-Gutiérrez et al., 2016, p. 244), que aplicará el criterio de Ward, puesto que generalmente es el que mejor se adapta a los objetivos de la investigación (Trespalacios-Gutiérrez et al., 2016, p. 244). En segundo lugar, aplicaremos Quick cluster, una técnica no jerárquica que, si bien presenta una estructura matemática menos precisa que la clasificación jerárquica (Valderrey, 2010, p. 364), nos servirá para valorar la robustez de la solución inicial propuesta. Este Quick cluster seguirá las recomendaciones de Trespalacios-Gutiérrez, Vázquez-Casielles y Bello-Acebrón (2005, p. 260) y Trespalacios-Gutiérrez et al. (2016, p. 247), esto es, que el número de grupos sea guiado por los resultados de los métodos jerárquicos. 
Tras mostrar las técnicas de análisis clúster a aplicar cuando el estudio descriptivo se muestre insuficiente, pasamos al siguiente apartado, que se dedica a exponer los principales resultados obtenidos en base al programa estadístico SPSS versión 23.

\section{Resultados}

Tabla 4. Ranking, items atendidos, calidad reutilizadora y puntuación total autonómica

\begin{tabular}{|c|c|c|c|c|c|c|c|c|c|c|c|c|}
\hline & & & & & c. 20 & & & c. 20 & & Dic. 2 & 17 , re & 2018 \\
\hline Rk1 & Rk2 & Rk3 & CCAA & Items & Cal. & PT13 & Items & Cal. & PT15 & Items & Cal. & PT18 \\
\hline $1^{\circ}$ & $1^{\circ}$ & $1^{\circ}$ & País Vasco & 11 & 33 & 44 & 12 & 36 & 48 & 10 & 30 & 40 \\
\hline $4^{\circ}-5^{\circ}$ & $5^{\circ}$ & $2^{\circ}$ & Andalucía & 5 & 11 & 16 & 5 & 11 & 16 & 7 & 21 & 28 \\
\hline $9 \circ$ & $9^{\circ}$ & $3^{\circ}$ & Cataluña & 2 & 4 & 6 & 2 & 4 & 6 & 5 & 20 & 25 \\
\hline $2^{\circ}$ & $2^{\circ}$ & $4^{\circ}-5^{\circ}$ & Com. Foral de Navarra & 6 & 18 & 24 & 6 & 18 & 24 & 6 & 18 & 24 \\
\hline $6^{\circ}$ & $6^{\circ}$ & $4^{\circ}-5^{\circ}$ & Castilla y León & 3 & 12 & 15 & 3 & 12 & 15 & 6 & 18 & 24 \\
\hline $4^{\circ}-5^{\circ}$ & $4^{\circ}$ & $6^{\circ}-7^{\circ}$ & Aragón & 3 & 9 & 12 & 4 & 12 & 16 & 5 & 15 & 20 \\
\hline $11^{\circ}-14^{\circ}$ & $11^{\circ}-17^{\circ}$ & $6^{\circ}-7^{\circ}$ & La Rioja & 0 & 0 & 0 & 0 & 0 & 0 & 5 & 15 & 20 \\
\hline 70 & 70 & $8^{\circ}$ & Islas Baleares & 2 & 7 & 9 & 2 & 7 & 9 & 2 & 8 & 10 \\
\hline $3^{\circ}$ & $3^{\circ}$ & $9^{\circ}$ & Extremadura & 5 & 15 & 20 & 5 & 15 & 20 & 2 & 6 & 8 \\
\hline $8^{\circ}$ & $8^{\circ}$ & $10^{\circ}$ & Canarias & 0 & 0 & 0 & 2 & 6 & 8 & 1 & 3 & 4 \\
\hline $10^{\circ}$ & $10^{\circ}$ & $11^{\circ}$ & Castilla-La Mancha & 0 & 0 & 0 & 1 & 4 & 5 & 1 & 3 & 4 \\
\hline $11^{\circ}-17^{\circ}$ & $11^{\circ}-17^{\circ}$ & $12^{\circ}$ & Región de Murcia & 0 & 0 & 0 & 0 & 0 & 0 & 1 & 2 & 3 \\
\hline $11^{\circ}-17^{\circ}$ & $11^{\circ}-17^{\circ}$ & $13^{\circ}-17^{\circ}$ & Cantabria & 0 & 0 & 0 & 0 & 0 & 0 & 0 & 0 & 0 \\
\hline $11^{\circ}-17^{\circ}$ & $11^{\circ}-17^{\circ}$ & $13^{\circ}-17^{\circ}$ & Comunidad de Madrid & 0 & 0 & 0 & 0 & 0 & 0 & 0 & 0 & 0 \\
\hline $11^{\circ}-14^{\circ}$ & $11^{\circ}-17^{\circ}$ & $13^{\circ}-17^{\circ}$ & Comunidad Valenciana & 0 & 0 & 0 & 0 & 0 & 0 & 0 & 0 & 0 \\
\hline $11^{\circ}-14^{\circ}$ & $11^{\circ}-17^{\circ}$ & $13^{\circ}-17^{\circ}$ & Galicia & 0 & 0 & 0 & 0 & 0 & 0 & 0 & 0 & 0 \\
\hline $11^{\circ}-14^{\circ}$ & $11^{\circ}-17^{\circ}$ & $13^{\circ}-17^{\circ}$ & Principado de Asturias & 0 & 0 & $\mathbf{0}$ & 0 & 0 & 0 & 0 & 0 & 0 \\
\hline \multicolumn{6}{|c|}{ Puntuación total } & 146 & & & 167 & & & 210 \\
\hline
\end{tabular}

El análisis descriptivo comienza (tabla 4) mostrando las puntuaciones que alcanza cada una de las CCAA para el indicador TOD tanto en diciembre de 2013 (apertura masiva de portales de datos abiertos), como en diciembre de 2015 (entrada en vigor de la Ley de transparencia, acceso a la información pública y buen gobierno para las entidades locales y autonómicas) y para 2017-2018 (última visita realizada ${ }^{9}$ ).

A primera vista puede apreciarse que el número de autonomías calificadas con cero puntos disminuye desde las ocho existentes en 2013, a siete en 2015 y a cinco en 2018. Otra noticia positiva es que la puntuación global aumenta desde los 146 puntos de 2013, a los 167 de 2015, alcanzando los 210 puntos en 2018. Esta mejora se debe a que son más las autonomías que experimentan progresos (mención especial para La Rioja y Cataluña) que las que se estancan o empeoran (destacando negativamente Extremadura que no actualiza la información buscada).

La tabla 5 ofrece un análisis desagregado del indicador por dimensiones. Todas las categorías presentan un desempeño limitado, si bien "gasto" presenta el mayor porcentaje de atención en todos los años de estudio, superando el $25 \%$ de la puntuación máxima aplicable (aunque tan solo en la revisión de 2018). No obstante, la evolución de todos los apartados es positiva con mejorías que oscilan entre el $35 \%$ y el $45 \%$, lo que refleja una consolidación longitudinal de las iniciativas.

El análisis por tipos, un importante cometido de esta investigación, se realiza de forma detallada para cada uno de los períodos en que la información fue recogida. El estudio comienza con un diagrama de barras que representa gráficamente la puntuación alcanzada por cada autonomía, lo que permite tener una sencilla valoración visual y finaliza, cuando se considera como necesario, con un análisis clúster.
En 2017, las diferencias interautonómicas se reducen, aunque el análisis clúster sigue identificando dos grupos de comunidades autónomas bien delimitados
Las puntuaciones alcanzadas por el indicador de transparencia datos abiertos son bajas en todos los períodos, si bien se aprecia una evolución positiva 
Tabla 5. Valoración y evolución de las dimensiones del indicador

\begin{tabular}{|c|c|c|c|c|c|c|c|c|c|c|c|c|}
\hline \multirow[b]{2}{*}{ CCAA } & \multicolumn{4}{|c|}{ Dic. 2013} & \multicolumn{4}{|c|}{ Dic. 15} & \multicolumn{4}{|c|}{ Dic. 2017, rev. 2018} \\
\hline & Ec-Fa & Gober. & Gasto & PT13 & Ec-Fa & Gober. & Gasto & PT15 & Ec-Fa & Gober. & Gasto & PT18 \\
\hline País Vasco & 20 & 10 & 14 & 44 & 24 & 10 & 14 & 48 & 17 & 15 & 8 & 40 \\
\hline Andalucía & 0 & 8 & 8 & 16 & 0 & 8 & 8 & 16 & 4 & 14 & 10 & 28 \\
\hline Cataluña & 6 & 0 & 0 & 6 & 6 & 0 & 0 & 6 & 15 & 0 & 5 & 25 \\
\hline Com. Foral de Navarra & 16 & 8 & 0 & 24 & 16 & 8 & 0 & 24 & 16 & 8 & 0 & 24 \\
\hline Castilla y León & 0 & 5 & 10 & 15 & 0 & 5 & 10 & 15 & 8 & 4 & 12 & 24 \\
\hline Aragón & 8 & 0 & 4 & 12 & 12 & 0 & 4 & 16 & 8 & 0 & 12 & 20 \\
\hline La Rioja & 0 & 0 & 0 & 0 & 0 & 0 & 0 & 0 & 8 & 0 & 12 & 20 \\
\hline Islas Baleares & 0 & 5 & 4 & 9 & 0 & 5 & 4 & 9 & 0 & 5 & 5 & 10 \\
\hline Extremadura & 8 & 4 & 8 & 20 & 8 & 4 & 8 & 20 & 8 & 0 & 0 & 8 \\
\hline Canarias & 0 & 0 & 0 & 0 & 0 & 8 & 0 & 8 & 0 & 4 & 0 & 4 \\
\hline Castilla-La Mancha & 0 & 0 & 0 & 0 & 0 & 5 & 0 & 5 & 0 & 4 & 0 & 4 \\
\hline Región de Murcia & 0 & 0 & 0 & 0 & 0 & 0 & 0 & $\mathbf{0}$ & 0 & 0 & 3 & 3 \\
\hline Cantabria & 0 & 0 & 0 & 0 & 0 & 0 & 0 & 0 & 0 & 0 & 0 & 0 \\
\hline Comunidad de Madrid & 0 & 0 & 0 & 0 & 0 & 0 & 0 & 0 & 0 & 0 & 0 & $\mathbf{0}$ \\
\hline Comunidad Valenciana & 0 & 0 & 0 & 0 & 0 & 0 & 0 & 0 & 0 & 0 & 0 & 0 \\
\hline Galicia & 0 & 0 & 0 & 0 & 0 & 0 & 0 & 0 & 0 & 0 & 0 & 0 \\
\hline Principado de Asturias & 0 & 0 & 0 & 0 & 0 & 0 & 0 & 0 & 0 & 0 & 0 & 0 \\
\hline Totales & 58 & 40 & 48 & 146 & 66 & 53 & 48 & 167 & 84 & 54 & 67 & 210 \\
\hline Puntuación máxima & 1.020 & 425 & 255 & 1.700 & 1.020 & 425 & 255 & 1.700 & 1.020 & 425 & 255 & 1.700 \\
\hline$\%$ atendido & 5,69 & 9,41 & 18,82 & 8,59 & 6,47 & 12,47 & 18,82 & 9,82 & 8,24 & 12,71 & 26,27 & 12,35 \\
\hline \multicolumn{5}{|l|}{$\%$ mejora bienal } & 13,79 & 32,50 & 0,00 & 14,38 & 27,27 & 1,89 & 39,58 & 25,75 \\
\hline \multicolumn{9}{|l|}{$\%$ mejora acumulada } & 44,83 & 35,00 & 39,58 & 43,84 \\
\hline
\end{tabular}

\section{Año 2013}

La observación de la figura 3 muestra como evidente la existencia de dos grupos de CCAA en 2013: autonomías con cero puntos versus autonomías con puntuación positiva, por lo que no parece necesario realizar un análisis de conglomerados.

Así pues, proponemos la existencia de dos conglomerados, indicando la asignación autonómica a continuación. Nueve CCAA pertenecerían al primer grupo, en el que tendríamos, por un lado, Canarias, Cantabria, Comunidad de Madrid, Comunidad Valenciana, Principado de Asturias y Región de Murcia, sin portal autonómico; y por otro, Castilla-La Mancha, Galicia y La Rioja, puesto que su repositorio no incorpora conjuntos de datos relacionados con nuestra definición de transparencia. El segundo grupo, el de las CCAA de divulgación más avanzada, quedaría compuesto por las ocho autonomías restantes, liderando de forma destacada el País Vasco.

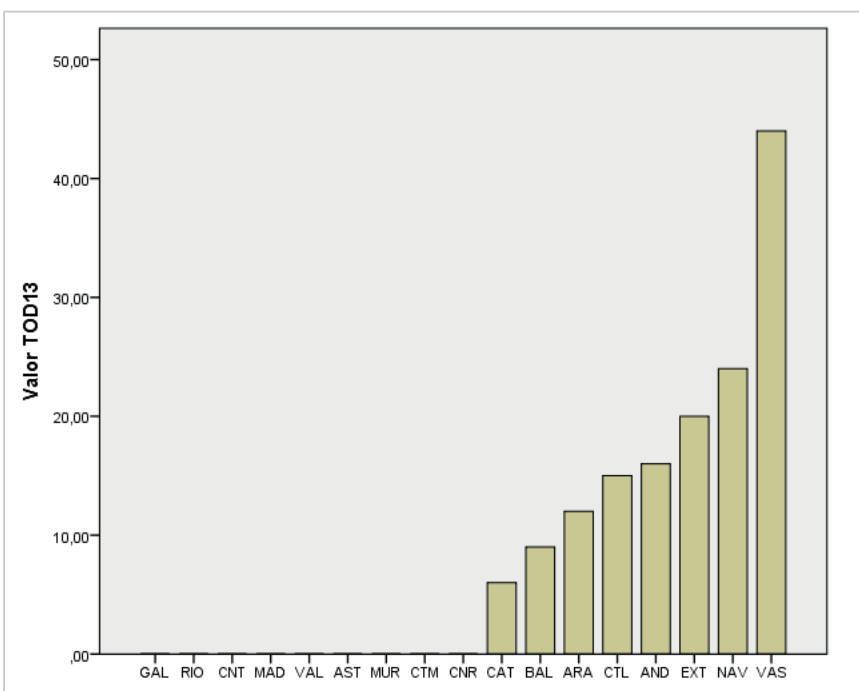

Figura 3. Puntuación del indicador de transparencia de datos abiertos en 2013

\section{Año 2015}

El indicador de 2015 refleja los cambios en los dos años transcurridos. Apreciamos que:

- Canarias, Comunidad Valenciana y Asturias se han incorporado a la iniciativa de portales datos abiertos autonómicos;

- los conjuntos de datos relacionados con la transparencia han aumentado ligeramente.

A nivel global, los avances son escasos, lo que se traduce en la práctica ausencia de cambios en el ranking, dando a entender que la entrada en vigor de la Ley 19/2013 apenas ha tenido impacto en la divulgación de datos para la rendición de cuentas. Además, la división propuesta también es en dos grupos de autonomías (figura 4). 
Al primer conglomerado pertenecería el $40 \%$ de las CCAA que, o bien no tienen portal (Comunidad de Madrid, Región de Murcia y Cantabria) o bien no publican información que permita completar ningún ítem (Galicia, Comunidad Valenciana, Principado de Asturias y La Rioja). Al segundo grupo pertenecerían las diez CCAA con puntuaciones positivas, entre las cuales sigue destacando el País Vasco, que mantiene el primer puesto de forma holgada.

\section{Año 2018}

Los portales de datos abiertos experimentan avances en cuanto a la divulgación de información relacionada con la rendición de cuentas, apreciando una mejoría que duplica a la producida en los dos años anteriores (la mejora en este último período es de 43 puntos, mientras que la experimentada entre 2013-2015 es de tan sólo 19 puntos). La figura 5 muestra los valores del indicador.

Tan sólo permanecen cinco CCAA valoradas con cero puntos por el indicador, un porcentaje inferior al 30\%: Comunidad de Madrid y Cantabria, sin portal de datos de uso operativo; y Galicia, Comunidad Valenciana y Principado de Asturias, que siguen sin ofrecer conjuntos de datos transparentes. Otro grupo de cinco autonomías presenta una valoración exigua (Región de Murcia, Castilla-La Mancha, Canarias, Extremadura y Baleares) oscilando entre los tres y los diez puntos TOD. A partir de aquí, y con un significativo salto de diez puntos, se encuentran las autonomías más avanzadas: La Rioja, Aragón, Castilla y León, Comunidad Foral de Navarra, Cataluña y Andalucía, que han recortado la distancia frente al líder, el País Vasco, quien ha visto reducida levemente su valoración al no mantener actualizada toda la información disponible en revisiones anteriores.

A la luz de la observación de la figura 5, y en base a lo descrito, no parece posible realizar una discriminación sencilla entre autonomías. Por ello, decidimos acometer un análisis clúster, comenzando con el método jerárquico propuesto por Ward, para contrastar posteriormente la solución propuesta mediante el método no jerárquico de $K$ means, que ofrece como solución más plausible la existencia de dos grupos de CCAA.

La tabla 6, que recoge para cada uno de los años analizados la asignación de cada autonomía a las CCAA más avanzadas / rezagadas, da por finalizado este apartado con el que pasamos a señalar las conclusiones más relevantes de nuestro estudio.

\section{Conclusiones, limitaciones y futuras líneas de investigación}

Una vez puesto de manifiesto que los portales de datos abiertos se han consolidado a nivel mundial como una excelente iniciativa para que gobiernos e instituciones de todo tipo depositen sus datos de forma accesible, con licencia libre, y de forma no discriminatoria, favoreciendo por tanto su procesabilidad y

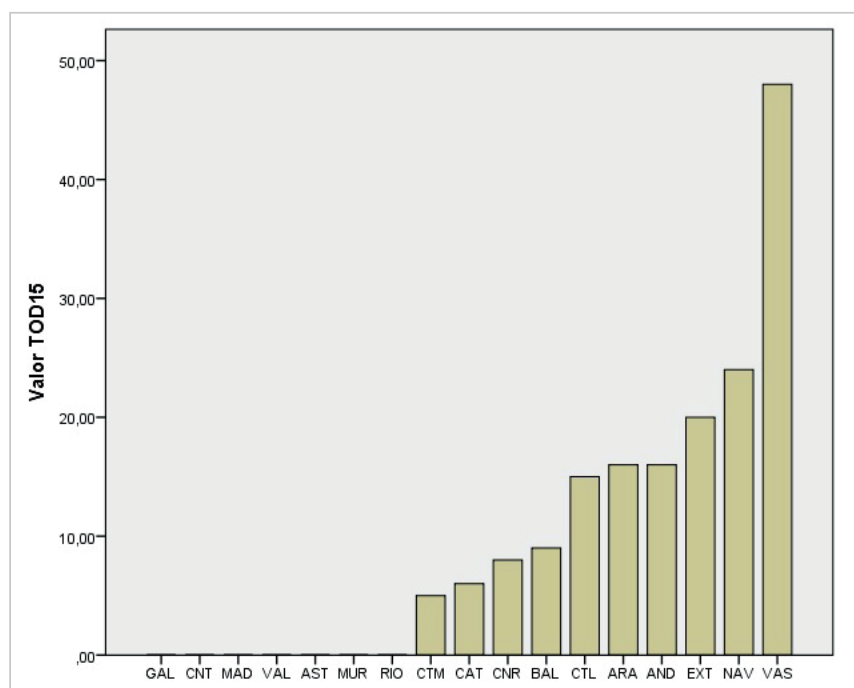

Figura 4. Puntuación del indicador de transparencia de datos abiertos en 2015

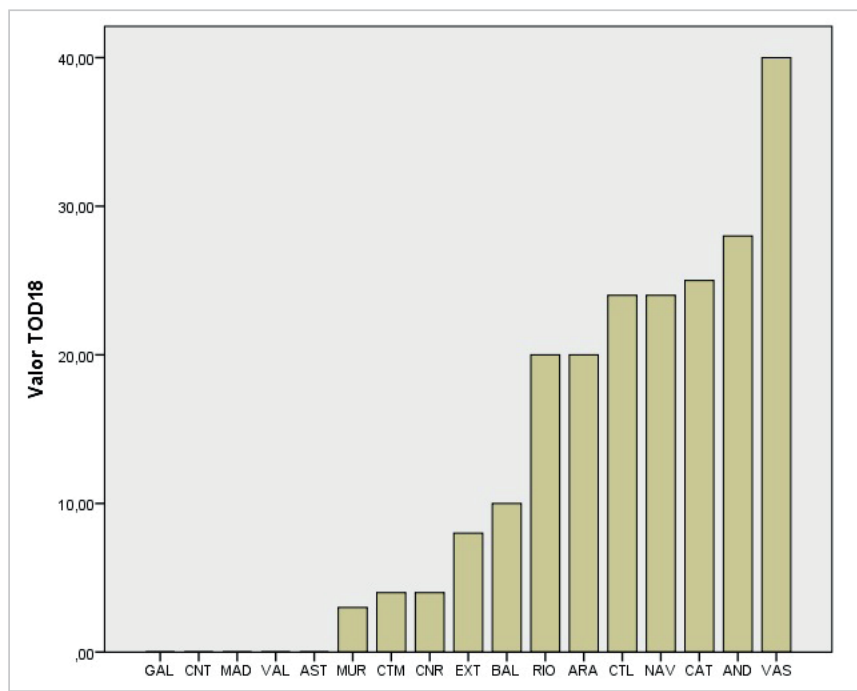

Figura 5. Puntuación del indicador de transparencia de datos abiertos en 2018

Tabla 6. Tipos y evolución de la transparencia activa de las CCAA según sus indicadores TOD

\begin{tabular}{|l|l|l|l|}
\hline \multicolumn{1}{|c|}{ Comunidad autónoma } & \multicolumn{1}{|c|}{$\mathbf{2 0 1 3}$} & \multicolumn{1}{c|}{$\mathbf{2 0 1 5}$} & \multicolumn{1}{c|}{$\mathbf{2 0 1 8}$} \\
\hline Andalucía & Avanzada & Avanzada & Avanzada \\
\hline Aragón & Avanzada & Avanzada & Avanzada \\
\hline Canarias & Rezagada & Avanzada & Rezagada \\
\hline Cantabria & Rezagada & Rezagada & Rezagada \\
\hline Castilla-La Mancha & Rezagada & Avanzada & Rezagada \\
\hline Castilla y León & Avanzada & Avanzada & Avanzada \\
\hline Cataluña & Avanzada & Avanzada & Avanzada \\
\hline Comunidad de Madrid & Rezagada & Rezagada & Rezagada \\
\hline Comunidad Foral de Navarra & Avanzada & Avanzada & Avanzada \\
\hline Comunidad Valenciana & Rezagada & Rezagada & Rezagada \\
\hline Extremadura & Avanzada & Avanzada & Rezagada \\
\hline Galicia & Rezagada & Rezagada & Rezagada \\
\hline Islas Baleares & Avanzada & Avanzada & Rezagada \\
\hline La Rioja & Rezagada & Rezagada & Avanzada \\
\hline País Vasco & Avanzada & Avanzada & Avanzada \\
\hline Principado de Asturias & Rezagada & Rezagada & Rezagada \\
\hline Región de Murcia & Rezagada & Rezagada & Rezagada \\
\hline
\end{tabular}


consecuentemente su reutilización, hemos apreciado que nuestro país se ha sumado exitosamente a este este movimiento. Por ello, nos ha parecido interesante, debido a la importancia de las comunidades autónomas en nuestro país, enfocar el estudio al entorno de los portales regionales analizando el grado de atención que prestan a un objetivo: el fomento de la transparencia.
La asignación de cada autonomía al conglomerado de transparencia avanzada o transparencia rezagada se mantiene bastante estable a lo largo de todo el estudio

Si bien es cierto que la transparencia tiene varios componentes, hemos decidido centrarnos en la divulgación de información de forma proactiva, siendo futuros estudios los que incorporen otras vertientes, como el grado de atención a las solicitudes de acceso a la información. A estas consideraciones hay que añadir que entendemos esta divulgación en formatos reutilizables no obligatoria, según lo mencionado por la Ley 19/2013 y recomendable, según indica la Ley 8/2015, de 9 de julio, por la que se modifica la Ley 37/2007, de 16 de noviembre, sobre reutilización de la información del sector público, que señala que se procurará siempre que sea posible proporcionar la información en formato abierto y legible por máquina, conjuntamente con sus metadatos y con los niveles más elevados de precisión y desagregación.

La transparencia activa ha sido medida en base a una lista de comprobación propia compuesta por 20 items, motivada por lo que sería interesante encontrar disponible tras la lectura del capítulo de transparencia activa de la Ley 19/2013 de transparencia, acceso a la información pública y buen gobierno, básicamente en sus artículos 6, información institucional, organizativa y de planificación; y 8 , información económica, presupuestaria y estadística. Tras ponderar la existencia de información en función de su capacidad reutilizadora según lo propuesto por Tim Berners-Lee, quedó formulado nuestro indicador de transparencia datos abiertos calculado en tres períodos de tiempo, 2013, 2015 y 2018.

Este indicador ofreció unos resultados mediocres, puesto que las puntuaciones medias son muy bajas $(8,6,9,8$ y de 12,35 respectivamente en cada período), lo que supone que apenas se completa el 10\% de la valoración total máxima, y eso sólo para la revisión más reciente. Esto equivale a afirmar que, salvo determinadas excepciones, se está desatendiendo el potencial de los datos abiertos para la rendición de cuentas.

Por su parte, el análisis deja interesantes conclusiones:

- se aprecia una polarización muy marcada en los años 2013 y 2015, que permite una discriminación sencilla a nivel regional entre las comunidades que se incorporan a la iniciativa y publican algo de información transparente y las que o bien carecen de portal o no divulgan absolutamente nada;

- para 2018 las diferencias se ven suavizadas, lo que nos invita a realizar un análisis de conglomerados que asegure la imparcialidad y minimice el riesgo de equívocos.

Siguiendo las recomendaciones de combinar más de un método de análisis clúster, y para obtener una mayor garantía en el resultado, utilizamos modelos jerárquicos (Ward) y no jerárquicos ( $k$ means) que siguen permitiendo identificar dos grupos diferenciados, estableciéndose la frontera divisora en el salto de los 10 y 20 puntos.
Se aprecia una polarización casi dicotómica en la transparencia activa de las autonomías para las revisiones de 2013 y 2015

Estos hallazgos están en línea con las teorías sobre la divulgación de información del sector público de la agencia y neoinstitucional que postulan, respectivamente, la necesidad de publicar información que reduzca las asimetrías principal-agente o simplemente conformarse en imitar las iniciativas favorecedoras de una buena imagen pública, en un evidente fenómeno de isomorfismo institucional, pero sin incorporar información relevante para el control de su gestión.

La dicotomía hallada guarda relación con los conceptos de transparencia sincera aparente y transparencia aparente utilizados en otras investigaciones y que aquí se aplica a las autonomías avanzadas o rezagadas en divulgación, según su pertenencia a los equilibrados conglomerados obtenidos a lo largo del análisis longitudinal, que obviamente son susceptibles de cambios, al igual que lo hacen las puntuaciones del indicador en cada uno de los años.

Debe señalarse, no obstante, que prima la estabilidad en cuanto a la pertenencia autonómica a cada clúster. Doce comunidades no experimentan cambios: seis siempre han sido triple $A$, es decir siempre han estado en el grupo de las avanzadas (Andalucía, Aragón, Castilla y León, Cataluña, Comunidad Foral de Navarra y País Vasco) mientras que otras seis han sido siempre rezagadas (Cantabria, Comunidad de Madrid, Comunidad Valenciana, Galicia, Principado de Asturias y Región de Murcia). Únicamente experimentan variación Canarias y Castilla la Mancha, que sólo han estado mejor que la media en 2015; Extremadura, que mantiene su iniciativa prácticamente abandonada; y La Rioja que, tras dos períodos en el furgón de cola, está ahora junto a Aragón en el grupo de las mejores autonomías.

En cuanto a las limitaciones de la investigación, debemos mencionar la subjetividad de nuestra definición de transparencia, tanto en los items incorporados (que creemos, no obstante, de gran interés para la ciudadanía) como en la ponderación llevada a cabo. De todas formas, el uso del indicador propuesto ha permitido obtener una escala sencilla (de 0 a 100 puntos) y un rango de variabilidad en los resultados superior a otros como el Índice de transparencia de las CCAA, elaborado por Transparencia Internacional España (que carece de capacidad discriminadora entre regiones). En todo 
caso, y en línea con otros indicadores como Meloda, pensamos que en futuros estudios deben ser contempladas nuevas dimensiones de calidad reutilizadora, como las licencias de los conjuntos de datos. Igualmente debe considerarse el efecto de las solicitudes de información ciudadana yendo un paso más allá de la transparencia activa.

Nuestra reflexión final consiste en el deseo de que próximos estudios muestren mejores resultados en las puntuaciones y de que los dos grupos de autonomías converjan en uno solo, donde sólo se aprecie transparencia sincera en sus portales de datos abiertos, lo que significará que se estarán aprovechando las bondades de la divulgación de información transparente en formatos reutilizables.

\section{Notas}

1. No son portales autonómicos al uso. Se trata de proyectos de tipo estadístico que muestran la información desagregada, debiendo interactuar con los menús existentes para la generación de datos.

2. El portal se inauguró el 10 de abril de 2019 con 170 conjuntos de datos. Además, una revisión efectuada en mayo de 2019 de sus 172 conjuntos de datos no consiguió localizar ninguna información buscada por nuestro estudio, por lo que incluso si esta iniciativa hubiera sido tenida en consideración no afectaría a los resultados alcanzados.

3. Considerados como la aplicación de los datos abiertos al caso específico de la información que gestionan las administraciones públicas u otros organismos dependientes. Este manuscrito utiliza indistintamente los términos open data $u$ open government data puesto que sólo se ocupa de datos puestos a disposición por las administraciones públicas.

4. Transparencia y rendición de cuentas son términos relacionados pero no sinónimos. Rubiños-Gil (2014) señala que la transparencia tendría que ver con la idea de visibilizar el interior de una organización, mientras que la rendición de cuentas sería la necesidad de que ésta responda por su comportamiento o actividad, explicando cómo se gestionan los fondos públicos.

5. No es objetivo de este estudio ser excesivamente exhaustivo en las iniciativas favorecedoras. Una revisión más detallada se puede consultar en Curto-Rodríguez (2017).

6. Comienzo en Curto-Rodríguez (2015), siendo los últimos trabajos publicados los de García-García y Curto-Rodríguez (2018; 2019a; 2019b).

7. En https://5stardata.info se ofrece una detallada información de las características de cada nivel, así como sus costos y beneficios.

8. Formato/s nivel uno, un punto adicional; nivel dos, dos puntos extras; nivel tres, tres puntos, y resto de niveles, cuatro puntos adicionales. Hemos decidido, al igual que Fundación Orange (2014) fusionar los niveles 4 y 5, ambos muy relacionados con el concepto de la web semántica, representando el estadio superior en la reutilización de los datos.

9. En 2018 realizamos un muestreo de autonomías para evaluar la necesidad de realizar una nueva recogida. Ante la ausencia de cambios, mantuvimos los de diciembre de 2017 como actualizados.

\section{Referencias}

Abella, Alberto (2016). "Full description of Meloda". Meloda Portal for data publishers and profesional reusers of data. http://www.meloda.org/full-description-of-meloda

Abella, Alberto; Ortiz-de-Urbina-Criado, Maria; De-Pablos-Heredero, Carmen (2014). “Meloda, métrica para evaluar la reutilización de datos abiertos". El profesional de la información, v. 23, n. 6, pp. 582-588.

https://doi.org/10.3145/epi.2014.nov.04

Abella, Alberto; Ortiz-de-Urbina-Criado, María; De-Pablos-Heredero, Carmen (2018). "Indicadores de calidad de datos abiertos: el caso del portal de datos abiertos de Barcelona". El profesional de la información, v. 27, n. 2, pp. $375-382$. https://doi.org/10.3145/epi.2018.mar.16

Abu-Bakar, Nur-Barizah; Saleh, Zakiah (2011). "Incentives for disclosure of accounting information in public sector: a literature survey". International research journal of finance and economics, v. 75, pp. 24-38.

Alonso-Magdaleno, María-Lorena; García-García, Jesús (2014). “Evaluación de la transparencia municipal en el Principado de Asturias". Auditoría pública, v. 64, pp. 75-86.

http://asocex.es/evaluacion-de-la-transparencia-municipal-en-el-principado-de-asturias

Alt, James E.; Lassen, David-Dreyer; Rose, Shanna (2006). "The causes of fiscal transparency: Evidence from the US states". IMF staff papers, v. 53, n. 1, pp. 30-57.

https://doi.org/10.2307/30036021

Ballester-Espinosa, Adrián (2015). “Administración electrónica, transparencia y open data. Generadores de confianza en las Administraciones Públicas". Telos: Cuadernos de comunicación e innovación, v. 100, pp. 120-126.

https://telos.fundaciontelefonica.com/archivo/numero100/generadores-de-confianza-en-las-administraciones-publicas 
Bastida, Francisco; Benito, Bernardino; Guillamón, María-Dolores; Ríos, Ana-María (2019). “Tax mimicking in Spanish municipalities: expenditure spillovers, yardstick competition, or tax competition?”. Public sector economics, v. 43, n. 2, pp. 115-139. http://www.pse-journal.hr/en/archive/tax-mimicking-in-spanish-municipalities-expenditure-spillovers-yardstickcompetition-or-tax-competition_4110

Beltrán-Orenes, Pilar; Martínez-Pastor, Esther (2016). "Grado de cumplimiento de las Leyes de transparencia, acceso y buen gobierno y de reutilización de los datos de contratación de la administración central española". El profesional de la información, v. 25, n. 4, pp. 557-567.

https://doi.org/10.3145/epi.2016.jul.05

Beltrán-Orenes, Pilar; Martínez-Pastor, Esther (2017). “Organización de la información y sujetos obligados en el Portal de la transparencia de España”. El profesional de la información, v. 26, n. 5, pp. 983-994.

https://doi.org/10.3145/epi.2017.sep.19

Berners-Lee, Tim (2010). Linked data. World Wide Web Consortium. http://www.w3.org/Design/ssues/LinkedData.html

Bertot, John C.; Jaeger, Paul T.; Grimes, Justin M. (2010). "Using ICTs to create a culture of transparency: E-government and social media as openness and anticorruption tools for societies". Government information quarterly, v. 27, n. 3, pp. $264-271$. https://doi.org/10.1016/j.giq.2010.03.001

Boix, Andrés (2015). "Transparencia, participación y procedimiento de elaboración de disposiciones reglamentarias para un modelo de open government". En: Cotino-Hueso, Lorenzo; Sahuquillo-Orozco, José-Luis; Corredoira-Alfonso, Loreto (eds.). El paradigma del gobierno abierto. Retos y oportunidades de la participación, transparencia y colaboración. Madrid: Universidad Complutense de Madrid, 123-129. ISBN: 9788460696780 https://eprints.ucm.es/35859/1/El_Paradigma_del_Gobierno..-Loreto\%20Corredoira.pdf

Cerrillo-Martínez, Agustí (2012). "La contribución de las TIC a la mejora de la transparencia administrativa". Arbor, n. 756, pp. 707-724. https://doi.org/10.3989/arbor.2012.756n4006

Cerrillo-Martínez, Agustí (2014). “Los principios de datos abiertos en la legislación española". Revista de internet, derecho y política, v. 19, pp. 62-77.

https://doi.org/10.7238/idp.v0i19.2418

Cerrillo-Martínez, Agustí (2018). "Datos masivos y datos abiertos para una gobernanza inteligente". El profesional de la información, v. 27, n. 5, pp. 1-10. https://doi.org/10.3145/epi.2018.sep.16

Cotino-Hueso, Lorenzo (2014). "La nueva Ley de transparencia y acceso a la información". Anuario de la Facultad de Derecho, n. 7, pp. 241-256. Universidad de Alcalá.

http://documentostics.com/component/option.com_docman/task,doc_view/gid,1543

Cotino-Hueso, Lorenzo (2015). "Derechos humanos, internet y TICs". En: Rey-Martínez, Fernando (dir.). Los derechos humanos en España: un balance crítico. Valencia: Tirant Lo Blanch, pp. 449-513. ISBN: 9788437097299

Cuadrado-Ballesteros, Beatriz (2014). "The impact of functional decentralization and externalization on local government transparency". Government information quarterly, n. 31, n. 2, pp. 265-277.

https://doi.org/10.1016/j.giq.2013.10.012

Curto-Rodríguez, Ricardo (2015). "Los portales de datos autonómicos y la rendición de cuentas". Auditoría pública, v. 66, pp. 75-83.

http://asocex.es/los-portales-de-datos-abiertos-autonomicos-y-la-rendicion-de-cuentas

Curto-Rodríguez, Ricardo (2016). "La información relacionada con la rendición de cuentas suministrada por las comunidades autónomas españolas". Auditoría pública, v. 67, pp. 61-68.

http://asocex.es/la-informacion-relacionada-con-la-rendicion-de-cuentas-suministrada-por-las-comunidades-autonomas-espanolas

Curto-Rodríguez, Ricardo (2017). Datos abiertos y rendición de cuentas en las comunidades autónomas españolas. Niveles de divulgación y determinantes. Tesis doctoral. Universidad de Oviedo, Departamento de Contabilidad. http://digibuo.uniovi.es/dspace/bitstream/10651/42797/1/TD_ricardocurto.pdf

Curto-Rodríguez, Ricardo (2019a). “Opciones de interacción, participación y colaboración en los portales de datos abiertos autonómicos españoles para el período 2014-2018”. Revista buen gobierno, v. 27, pp. 1-20.

Curto-Rodríguez, Ricardo (2019b). “¿Qué información relacionada con la publicidad activa contienen los portales de datos abiertos autonómicos españoles? Un análisis detallado tras cuatro años de su eclosión”. Revista española de transparencia, v. 9, pp. 177-198.

https://www.revistatransparencia.com/ojs/index.php/ret/article/view/48 
Delgado-Morales, Francisco (2018). “Derecho de acceso y brecha de género”. Revista española de transparencia, v. 6, pp. 23-31. https://drive.google.com/file/d/1RG-mykY8GOlxmQzpgpN9Muhzh3vZiCO-/view

Delgado-Morales, Francisco; López-Carvajal, Francisco-Javier; Sierra-Rodríguez, Javier (2015). “Regulación y sistemas de evaluación de la Transparencia en España". Revista de derecho de la Hacienda Pública, v. 5, pp. 111-134.

https://cgrfiles.cgr.go.cr/publico/docsweb/documentos/publicaciones-cgr/revista-derecho/05/revista-derecho-05.pdf

European Commission (2014). IEAG. UN Secretary-General's independent expert advisory group on the data revolution for sustainable development.

https://ec.europa.eu/knowledge4policy/organisation/ieag-un-secretary-general\%E2\%80\%99s-independent-expertadvisory-group-data-revolution_en

European Data Portal (2018). Open data best practices in Europe's top three performers.

https://www.europeandataportal.eu/es/highlights/open-data-maturity-2018-report\%E2\%80\%99s-top-three-performers

FEMP (2019). Datos abiertos FEMP 2019. 40 conjuntos de datos a publicar por las entidades locales. Federación Española de Municipios y Provincias.

http://femp.femp.es/files/3580-1938-fichero/DATOS\%20ABIERTOS\%20FEMP\%202019.pdf

Ferejohn, John (1999). "Accountability and authority: toward a theory of political accountability”. In: Przeworski, Adam; Stokes, Susan C.; Manin, Bernard. Democracy, accountability, and representation. Cambridge University Press, pp. 131-133. https://doi.org/10.1017/CB09781139175104.005

Fernández-Llera, Roberto; Morán-Méndez, Eloy (2013). “Las comunidades autónomas en la crisis: cifras, planes y retos de control". Auditoría pública: revista de los órganos autónomos de control externo, v. 59, pp. 39-50.

http://asocex.es/las-comunidades-autonomas-en-la-crisis-cifras-planes-y-retos-de-control

Ferrer-Sapena, Antonia; Peset, Fernanda; Aleixandre-Benavent, Rafael (2011). “Acceso a los datos públicos y su reutilización: open data y open government". El profesional de la información, v. 20, n. 3, pp. 260-269.

https://doi.org/10.3145/epi.2011.may.03

Ferrer-Sapena, Antonia; Sánchez-Pérez, Enrique A. (2013). “Open data, big data: ¿'hacia dónde nos dirigimos?”. Anuario ThinkEPI, v. 7, pp. 150-156.

https://recyt.fecyt.es/index.php/ThinkEPI/article/view/30351

Fundación Orange (2014). Datos abiertos en las comunidades autónomas y sus mayores ayuntamientos 2014. Fundación Orange; Arvo Consultores y Tecnología.

https://www.proyectosfundacionorange.es/docs/eE2014/Datos_Abiertos_2014.pdf

Galli, Enma; Rizzo, Ilde; Scaglioni, Carla (2019). "Transparency and socio-political environment in Italy". In: Bianco, Adele; Conigliaro, Paola; Gnaldi Michela (eds.). Italian studies on quality of life. Social indicators research series, pp. 97-109. Springer, Cham.

https://doi.org/10.1007/978-3-030-06022-0_7

García-García, Alicia (2013). Datos abiertos enlazados, linked open data en documentación científica. Tesis de master. Universidad Politécnica de Valencia, Departamento de Comunicación Audivisual, Documentación e Historia del Arte.

https://riunet.upv.es/handle/10251/18272

García-García, Jesús; Alonso-Magdaleno, María-Isabel; Alonso-Magdaleno, María-Lorena (2016). “Determinantes de la transparencia en municipios de mediano y pequeño tamaño". Auditoría pública: revista de los órganos autónomos de control externo, v. 67, pp. 51-60.

http://asocex.es/determinantes-de-la-transparencia-en-municipios-de-mediano-y-pequeno-tamano

García-García, Jesús; Curto-Rodríguez, Ricardo (2018). “Determinantes de la apertura de datos y rendición de cuentas en los gobiernos regionales españoles". Revista del CLAD reforma y democracia, v. 70, pp. 163-198.

http://old.clad.org/portal/publicaciones-del-clad/revista-clad-reforma-democracia/articulos/070-febrero-2018/Garcia-Curto.pdf

García-García, Jesús; Curto-Rodríguez, Ricardo (2019a). “Divulgación de información pública en las comunidades autónomas españolas (2013-2017): portal de datos abiertos, portal de transparencia y web institucional”. El profesional de la información, v. 27, n. 5, pp. 1051-1060.

https://doi.org/10.3145/epi.2018.sep.09

García-García, Jesús; Curto-Rodríguez, Ricardo (2019b). “El ejercicio de la rendición de cuentas mediante portales de datos abiertos en las comunidades autónomas españolas". IDP. Revista de internet, derecho y política, n. 29.

https://doi.org/10.7238/idp.v0i29.3182

Graells-Costa, Jordi (2013). “El arte de gobernar en abierto. Ideas y experiencias desde Cataluña”. En: Hofmann, Andrés; Ramírez-Alujas, Álvaro; Bojórquez-Pereznieto, José-Antonio (coords.). La promesa del gobierno abierto. México: ITAIP e InfoDF, pp. 461-489.

http://www.itaip.org.mx/slide/pdf/lpga.pdf 
Grande-Esteban, Ildefonso; Abascal-Fernández, Elena (2007). Fundamentos y técnicas de investigación comercial. 9ạ edición. Madrid: Esic Editorial. ISBN 9788473562881

Höchtl, Johann (2012). "Open government data - beyond the hype". In: CeDEM 12 Conference for e-democracy and open government. Danube-University Krems, Austria: Edition-Donau-Univ., pp. 363-366.

Laswad, Fawzi; Fisher, Richard; Oyelere, Peter (2005). “Determinants of voluntary internet financial reporting by local government authorities". Journal of accounting and public policy, v. 24, n. 2, pp. 101-121.

https://doi.org/10.1016/j.jaccpubpol.2004.12.006

Ma, Liang; Wu, Jiannan (2011). "What drives fiscal transparency? Evidence from provincial governments in China". In: $1^{\text {st }}$ Global conference on transparency research, Rutgers University-Newark, May 19-20.

Martínez-Moya, Diego (2015). “Evaluación de los aspectos de usabilidad y reutilización de información en el Portal de la Transparencia de España”. Cuadernos de gestión de información, v. 5, n. 1, pp. 36-52.

https://revistas.um.es/gesinfo/article/view/232211

Martínez-Vázquez, Jorge (2014). "La descentralización tributaria a las comunidades autónomas en España: desafíos y soluciones". En: Esteller-Moré, Alejandro; Duran-Cabré, José-María (eds.). Por una verdadera reforma fiscal. Editorial Ariel. ISBN: 9788408123668

Open Data Soft (2019). Open data inception.

https://opendatainception.io/\#

Rodríguez-Bolívar, Manuel-Pedro; Alcaide-Muñoz, Laura; López-Hernández, Antonio M. (2013). “Determinants of financial transparency in government". International public management journal, v. 16, pp. 557-602.

https://doi.org/10.1080/10967494.2013.849169

Rubiños-Gil, María (2014). "La transparencia, la comunicación y la confianza en las instituciones públicas: los casos comparados de la Freedom of Information Act en el Reino Unido y la Ley de Transparencia, Buen Gobierno y Acceso a la Información en España". En: III Congreso Internacional de Comunicación Política y Estrategias de Campaña. Santiago de Compostela. http://www.alicecomunicacionpolitica.com/files/ponencias/461-F54168fa34611410764707-ponencia-1.pdf

Sierra-Rodríguez, Javier (2018a). “A modo de introducción: apuntes básicos sobre la ley de transparencia, acceso a la información pública y buen gobierno". En: Sánchez-de-Diego-Fernández-de-la Riva, Manuel (coord.). Apuntes sobre la transparencia. Madrid: Universidad Complutense, pp. 13-32.

https://drive.google.com/file/d/1XJhnwrOLNhbEnvZQNIooTUt06NeXI3xe/view

Sierra-Rodríguez, Javier (2018b). "Una crítica al diseño de los organismos de garantía del derecho de acceso en las comunidades autónomas". Revista jurídica de Castilla y León, n. 45, pp. 73-112.

https://dialnet.unirioja.es/servlet/articulo?codigo=6392729

Sunlight Foundation (2007). Eight principles of open government data.

https://opengovdata.org

Surowiecki, James (2005). The wisdom of crowds. New York: Anchor Books. ISBN: 9780385721707

Trespalacios-Gutiérrez, Juan-Antonio; Vázquez-Casielles, Rodolfo; Bello-Acebrón, Laurentino (2005). Investigación de mercados: Métodos de recogida y análisis de la información para la toma de decisiones en marketing. Editorial Paraninfo. ISBN: 9788497323772

Trespalacios-Gutiérrez, Juan-Antonio; Vázquez-Casielles, Rodolfo; De-la-Ballina-Ballina, Francisco-Javier; Suárez-Vázquez, Ana (2016). Investigación de mercados: El valor de los estudios de mercado en la era del marketing digital. Editorial Paraninfo. ISBN: 9788428338523

Valderrey-Sanz, Pablo (2010). SPSS 17. Extracción del conocimiento a partir del análisis de datos. Editorial RA-MA. ISBN: 9788478979929

Vicente-Paños, Adrián; Jordán-Alfonso, Aurea (2017). “Acceso a la información pública y su reutilización en las comunidades autónomas: evaluación de la reutilización de datos abiertos". El profesional de la información, v. 26, n. 3, pp. 381-391. https://doi.org/10.3145/epi.2017.may.04

Villoria, Manuel (2014). La publicidad activa en la Ley de transparencia, acceso a la información y buen gobierno: posibilidades e insuficiencias. Generalitat de Catalunya.

http://exteriors.gencat.cat/web/.content/transparencia/publicacions/gov_obert1/governobert_1_es.pdf

Walsh, Jo; Pollock, Rufus (2007). Open data and componentization. XTech 2007. http://assets.okfn.org/files/talks/xtech_2007

Zimmerman, Jerold L. (1977). "The municipal accounting maze: An analysis of political incentives". Journal of accounting research, v. 15, pp. 1107-144.

https://doi.org/10.2307/2490636 